\title{
The analgesic efficacy of intravenous lidocaine infusion after laparoscopic fundoplication: a prospective, randomized, double-blind, placebo-controlled trial
}

\author{
This article was published in the following Dove Press journal: \\ Local and Regional Anesthesia \\ 2 December 2016 \\ Number of times this article has been viewed
}

\author{
Gregory J Dale' \\ Stephanie Phillips ${ }^{2}$ \\ Gregory L Falk ${ }^{3}$ \\ 'Westmead Hospital Clinical School, \\ The University of Sydney, ${ }^{2}$ Sydney \\ Adventist Hospital Clinical School, \\ The University of Sydney, ${ }^{3}$ Concord \\ Clinical School, The University of \\ Sydney, Sydney, Australia
}

\begin{abstract}
This study aimed to determine if intravenous lidocaine infusion reduces postoperative pain intensity following laparoscopic fundoplication surgery and to also validate the safety of intravenous lidocaine at the dose tested. This was an equally randomized, double-blind, placebo-controlled, parallel-group, single center trial. Adult patients undergoing laparoscopic fundoplication were recruited. The intervention group received $1 \mathrm{mg} / \mathrm{kg}$ intravenous lidocaine bolus prior to induction of anesthesia, then an intravenous infusion at $2 \mathrm{mg} / \mathrm{kg} / \mathrm{h}$ for 24 hours. The primary outcome was pain, measured using a numeric rating scale for 30 hours postoperatively. Secondary outcomes were nausea and vomiting, opioid requirements, adverse events, serum lidocaine concentration, and length of hospital stay. The study was terminated after an interim analysis of 24 patients showed evidence of futility. There was no difference in postoperative pain scores (lidocaine versus control, mean \pm standard deviation) at rest ( $2.0 \pm 2.7$ vs $2.1 \pm 2.4$, $P=0.286)$ or with movement $(2.0 \pm 2.6$ vs $2.6 \pm 2.7, P=0.487)$. Three adverse events occurred in the lidocaine group ( $25 \%$ of patients). Intravenous lidocaine did not provide clinically significant analgesia to patients undergoing laparoscopic fundoplication. The serum lidocaine concentration of patients who experienced adverse events were within the therapeutic range. This trial cannot confirm the safety of intravenous lidocaine at the dose tested.
\end{abstract}

Keywords: analgesia, local anesthetics, intravenous infusions, pharmacokinetics

\section{Introduction}

Despite multimodal analgesia, severe postoperative pain is still experienced by many patients. Opioids are relatively contraindicated following laparoscopic fundoplication as they often cause nausea and vomiting. Postoperative vomiting places undue pressure on the repaired diaphragm and gastric wrap, risking early failure of the surgery. ${ }^{1}$ As such, patients undergoing laparoscopic fundoplication need effective, parenteral, nonopioid analgesia.

Lidocaine is an inexpensive and widely accessible local anesthetic that possesses analgesic, anti-inflammatory, and antihyperalgesic properties. ${ }^{2-4}$ While most commonly used in infiltration and for central neuraxial and peripheral nerve blocks, lidocaine can also be given intravenously (IV) to treat acute perioperative pain ${ }^{4}$ and chronic neuropathic pain. ${ }^{5}$ The current evidence for using IV lidocaine to treat perioperative pain is based on 45 clinical trials contributing to five systematic reviews. ${ }^{4,6-9}$ These studies found that IV lidocaine infusion in the perioperative period decreases pain intensity, opioid requirements, the duration of postoperative ileus, and opioid-related
Correspondence: Gregory J Dale Westmead Hospital, Cnr Hawkesbury Road and Darcy Road, Westmead, Sydney, NSW 2145, Australia Email gregory.dale@health.nsw.gov.au 
side-effects, such as postoperative nausea and vomiting. These studies concluded that further research is needed to determine the optimum dose, timing, and duration of infusion of lidocaine in this setting.

The effect of IV lidocaine infusion has not been studied in patients undergoing laparoscopic fundoplication surgery. This randomized controlled trial tested the primary hypothesis that IV lidocaine decreases postoperative pain intensity (at rest and during movement) after laparoscopic fundoplication. In order to verify the safety of IV lidocaine infusion for analgesia, the following secondary outcomes were measured: nausea and vomiting, opioid requirements, adverse events, serum lidocaine concentration, and length of hospital stay.

\section{Methods}

\section{Study population}

This was an equally randomized, double-blind, placebocontrolled, parallel-group, single-center trial conducted at the Sydney Adventist Hospital, Wahroonga, Sydney, Australia. Approval was received from the Adventist HealthCare Limited Human Research Ethics Committee (EC00141) and written informed consent was obtained from all patients. The study was registered with the Australian and New Zealand Clinical Trials Registry (ACTRN12613000440729).

All adults (age $>18$ years) undergoing laparoscopic fundoplication surgery by a single surgeon (GLF) were eligible to participate in the study. Exclusion criteria were allergies to local anesthetics, chronic use of analgesics or corticosteroids, impaired hepatic function (any single liver function test $\geq 20 \%$ normal reference range), epilepsy or other seizure disorder, severe cardiac failure (left ventricular ejection fraction $\leq 0.35$ ) or cardiac arrhythmias and pregnancy.

\section{Procedures}

General anesthesia was standardized using midazolam $2.5 \mathrm{mg}$ IV, effect-site target controlled infusions of propofol and remifentanil. Tracheal intubation was facilitated by rocuronium $1.2 \mathrm{mg} / \mathrm{kg}$, and the lungs were ventilated with $33 \%$ oxygen in air using a circle system.

Intraoperatively, all patients received IV granisetron $3 \mathrm{mg}$ and dexamethasone $8 \mathrm{mg}$ as prophylaxis against nausea and vomiting and parecoxib $40 \mathrm{mg}$ for analgesia. Postoperative analgesia was commenced with fentanyl $1 \mu \mathrm{g} \mathrm{kg}^{-1} \mathrm{IV}$ at the cessation of the remifentanil infusion. The diaphragmatic crura and port sites were infiltrated with $20 \mathrm{~mL}$ ropivacaine $0.2 \%$ by the surgeon. A patient-controlled analgesia (PCA) device administering IV fentanyl was provided $(10 \mu \mathrm{g} / \mathrm{mL}$, $10 \mu \mathrm{g}$ bolus, 5 minute lockout, no background) and PCA usage was recorded. Fentanyl PCA was discontinued if nausea was reported by the patient. Acetaminophen ( $1 \mathrm{~g} \mathrm{IV}$ every 6 hours) and indomethacin (100 mg per rectum every 12 hours) were administered to provide multi-modal analgesia. Rescue antiemetics (ondansetron $4 \mathrm{mg}$ sublingual and droperidol $0.5 \mathrm{mg} / \mathrm{kg} \mathrm{IV}$ ) were offered to any patient who experienced nausea or vomiting. Postoperatively, all patients had electrocardiogram monitoring via telemetry for 24 hours.

Patients were randomly assigned to study groups in fixed blocks of 12 using a computer-generated table of random numbers through the use of the randomization.com program. No stratification was used.

The patients in the intervention group received $1 \mathrm{mg} / \mathrm{kg}$ IV lidocaine bolus at induction, followed immediately by an infusion at $2 \mathrm{mg} / \mathrm{kg} / \mathrm{h}$ for 24 hours. The patients in the control group were treated likewise using $0.9 \%$ sodium chloride in a double-blind fashion.

Lidocaine was acquired as Xylocard ${ }^{\circledR} 500$ ampoules (AstraZeneca Pty Ltd, North Ryde, NSW, Australia), containing $500 \mathrm{mg}$ of lidocaine hydrochloride in $5 \mathrm{~mL}$ of water. Lidocaine study drug was made to a concentration of $0.5 \%$ $(5 \mathrm{mg} / \mathrm{mL})$ and supplied in $1000 \mathrm{~mL}$ flasks compatible with IMED Gemini (IMED Corporation, San Diego, CA, USA) infusion pumps and giving sets. The lidocaine and placebo study drug were visually identical. No patient, research nurse, investigator, or any other medical or nursing staff was aware of the treatment assignments for the duration of the study.

The randomization schedule was stored in a locked cupboard that was only accessible by the randomization authority (thus concealed from all care providers and other research personnel). When a patient was recruited into the study, the randomization authority would prepare the appropriate study drug. The study drug was given to the anesthetist accompanied by a sealed, opaque, tamper-proof envelope containing the treatment allocation. This envelope was kept in the patient file at all times in case serious adverse event required the knowledge of treatment allocation. Envelopes were examined at the completion of the trial to ensure that they were unopened.

The primary outcome of the study was postoperative pain. Pain was assessed using an 11-point numeric rating scale (NRS-11). The patients were asked to score their current pain on two occasions: at rest and on mobilization from supine to sitting upright. Pain scores were obtained every 4 hours, for 30 hours following commencement of the trial drug infusion. The 11-point numeric rating scale is a validated, sensitive tool for assessing postoperative pain intensity. ${ }^{10,11}$ All trial data were entered directly into the electronic medical record, which had been adapted for the study and mandated the completion of all parameters. 
Secondary outcomes were nausea and vomiting, opioid requirements, adverse events, serum lidocaine concentration, and length of hospital stay. Patients were asked if they experienced any nausea or vomiting in the preceding 4 hours when pain scores were obtained. Responses were recorded as "yes" or "no" and this outcome was treated as binary data. Opioid requirements were recorded as the dose of fentanyl delivered (rather than total demands/attempts for analgesia). Only adverse events that required the patient to be discontinued from the trial were interpreted. Venous blood samples were collected from all patients every 4 hours for the duration of the trial. Serum was immediately separated and frozen, then later analyzed to determine the serum lidocaine concentration-time profile for the dosage regimen used in this study. Length of hospital stay was recorded as the number of nights the patient was in hospital.

\section{Statistical analyses}

A priori sample size was estimated on the basis of an absolute reduction in pain score. A reduction of two in the NRS-11 was reported to be the amount needed to cause a clinically significant reduction in a person's experience of postoperative pain. ${ }^{12,13} \mathrm{~A}$ sample size of 18 patients in each group was calculated to be sufficient to detect a difference of two in the mean pain score, assuming a standard deviation of $\pm 2^{12}$ with a significance level $\alpha=0.05$ and a power of $90 \%$.

Interim analysis was planned when $33 \%$ and $66 \%$ of subjects had been recruited. The study would be stopped early when there was sufficient evidence to claim superiority (net benefit) or inferiority (net harm), or futility (little chance of achieving statistical significance) if the futility index was found to be $>0.8 .^{14}$

Statistical analyses were performed using IBM SPSS Statistics v21.0 (IBM Corporation). Sample size calculations and conditional power analyses were performed using PASS v13 (NCSS, LLC). The comparison between the intervention and control groups was conducted using an independent samples $t$-test, Mann-Whitney-Wilcoxon $U$-test, chi-squared test, or Fisher's exact test as appropriate. Normally distributed data are presented as mean \pm standard deviation, nonnormally distributed data are presented as medians (interquartile range), and categorical data are presented as raw data and as frequencies. All statistical tests are two-sided with significance level $\alpha=0.05$. Unblinding was performed prior to the statistical analysis of data.

\section{Results}

Twenty-four patients entered the study and were randomized equally to two groups. Twelve patients $(100 \%)$ in the control group completed the study. Nine patients (75\%) in the intervention group completed the study. The three patients who did not complete the study were withdrawn due to adverse events suspicious of lidocaine toxicity (treatment allocation was not known until after withdrawal from the trial). The participant flow diagram is presented (Figure 1). A planned interim analysis was performed when recruitment was $66 \%$ complete. At this point, the trial was stopped early on the basis of futility, prior to reaching the target sample size of 36 patients (18 per group). The futility index was $99 \%$, that is, there was a $1 \%$ chance of rejecting a false null hypothesis at the end of the study given the data that had emerged. ${ }^{15}$

The demographic and clinical characteristics of both groups were similar (Table 1). There was no statistically significant difference in pain scores between treatment groups either at rest or during mobilization when summarized over the 30-hour postoperative period. At rest, the mean pain score was $2.0 \pm 2.7$ in the lidocaine group and $2.1 \pm 2.4$ in the control group $(P=0.286)$. With movement, the mean pain score was $2.0 \pm 2.6$ in the lidocaine group and $2.6 \pm$ 2.7 in the control group $(P=0.487)$. Box and whisker plots are presented for pain scores at rest (Figure 2) and during mobilization (Figure 3) for lidocaine and control groups stratified at 6 -hour intervals over the 30 -hour postoperative period.

The incidence of nausea in the lidocaine group (50\%) was similar to that in the control group (33\%) $(P=0.408)$. Three patients $(25 \%)$ in the lidocaine group vomited, whereas one patient ( $8 \%)$ in the control group vomited $(P=0.273)$.

The average total dose of fentanyl administered was $124 \pm 165 \mu \mathrm{g}$ for the lidocaine group and $344 \pm 426 \mu \mathrm{g}$ for the control group over the 30 -hour postoperative period $(P=0.117)$.

There were three clinically significant adverse events in the lidocaine group ( $25 \%$ of patients) compared with none in the control group, however, this difference was not statistically significant $(P=0.064)$. One adverse event, severe bradycardia causing loss of consciousness, responded rapidly to external cardiac massage. The other adverse events were neurological symptoms (perioral paresthesia and restless legs) that are associated with early local anesthetic toxicity. The maximum serum lidocaine concentrations in these patients were $4.3,2.7$, and $3.5 \mathrm{mg} / \mathrm{L}$.

The serum concentration-time profile of 12 patients who received the IV lidocaine infusion is presented in Figure 4. Two patients (22\%) had peak serum lidocaine concentration $\geq 5 \mathrm{mg} / \mathrm{L}(5.2$ and $5.0 \mathrm{mg} / \mathrm{L})$. Neither of these patients experienced clinical signs or symptoms of lidocaine toxicity. 


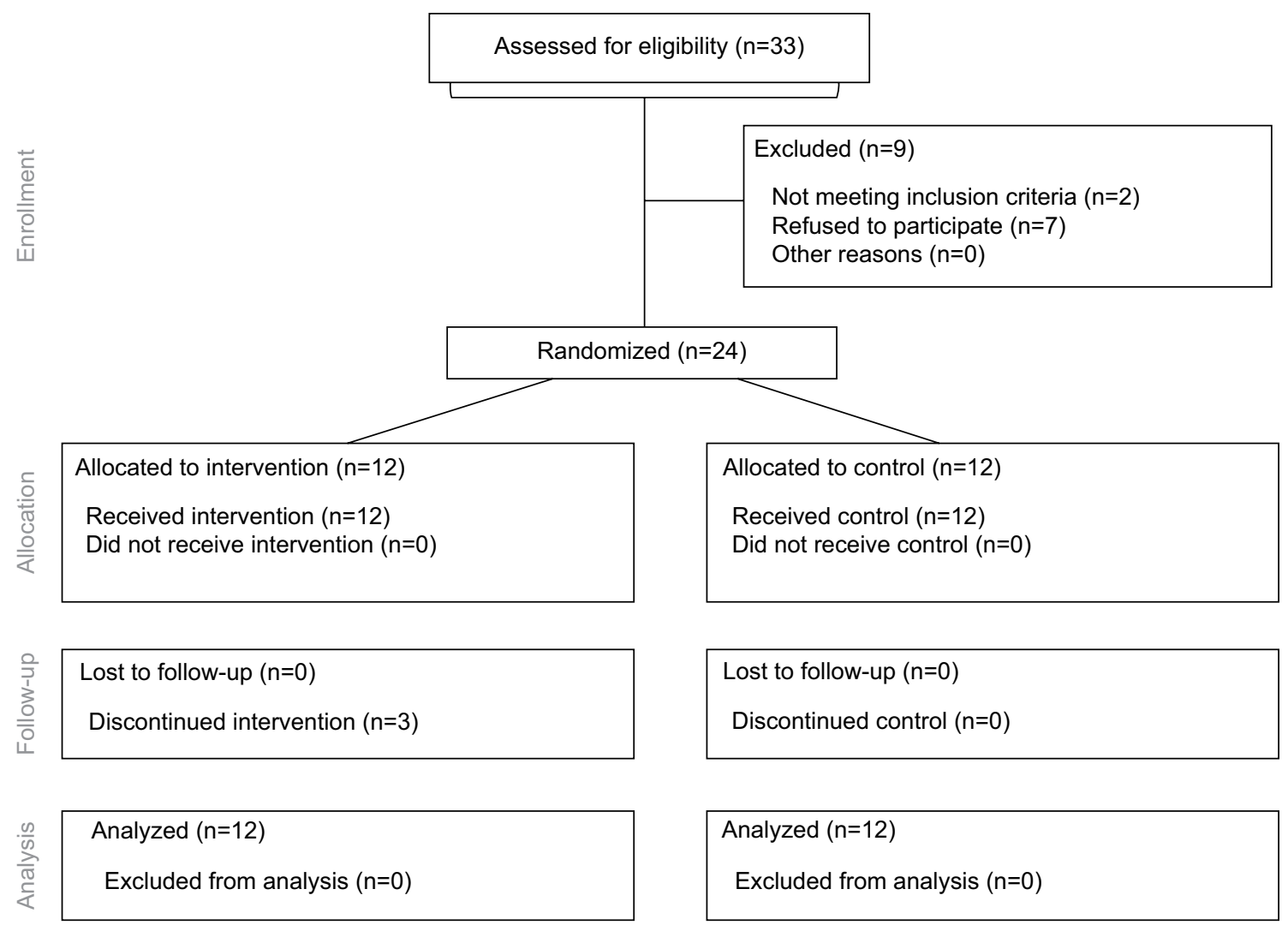

Figure I The CONSORT methods diagram showing participant flow. Abbreviation: CONSORT, consolidated standards of reporting trials.

Table I Characteristics of patients undergoing laparoscopic fundoplication

\begin{tabular}{lll}
\hline & Lidocaine $(\mathbf{n}=\mathbf{I} 2)$ & Control $(\mathbf{n}=\mathbf{I 2})$ \\
\hline Age (years) & $68.5(10.17)$ & $66.5(1 \mathrm{I} .39)$ \\
Sex (female) & $9(75 \%)$ & $6(50 \%)$ \\
Smoking status (current) & $1(8.33 \%)$ & $1(8.33 \%)$ \\
Height $(\mathrm{cm})$ & $165.75(9.76)$ & $165.33(10.69)$ \\
Body mass index $\left(\mathrm{kg} / \mathrm{m}^{2}\right)$ & $27.05(2.39)$ & $28.91(4.26)$ \\
Alcohol consumption $(\mathrm{g} /$ week) & $77(83.40)$ & $68(\mathrm{I} 20.06)$ \\
ASA status* & $2.33(0.49)$ & $2.27(0.47)$ \\
Stomach herniated $(\%)$ & $0.40(0.28)$ & $0.2 \mathrm{I}(0.27)$ \\
Surgery time $(\mathrm{min})$ & $68.83(20.76)$ & $64.50(19.58)$ \\
Remifentanil used $(\mu \mathrm{g} / \mathrm{kg} / \mathrm{h})$ & $5.33(2.16)$ & $4.79(\mathrm{I} .70)$ \\
Propofol used $(\mathrm{mg} / \mathrm{kg} / \mathrm{h})$ & $0.445(0.187)$ & $0.46 \mathrm{I}(0.109)$ \\
\hline
\end{tabular}

Notes: *Physical status score. Values are mean (SD) or number (proportion). Abbreviations: ASA, American Association of Anesthesiologists; SD, standard deviation.

There was no statistically significant difference in the mean length of hospital stay between groups; lidocaine group 2.5 days (95\% confidence interval $[\mathrm{CI}] 2.07-2.93$ ) and control group 2.25 days (95\% CI 1.96-2.54).

\section{Discussion}

This study used a high dose and long duration of intravenous lidocaine infusion in an attempt to identify a concentrationor time-dependent analgesic effect. Despite this, a clinically significant benefit of lidocaine was not demonstrated. This study cannot reject the possibility that a small amount of analgesia is achieved with intravenous lidocaine. A mean reduction of 0.54 ( $95 \% \mathrm{CI}:-1.56,0.48)$ in pain score during mobilization was found in this study. This effect size is similar to that reported in the meta-analysis, ${ }^{4}$ which showed intravenous lidocaine to reduce pain scores by 1.05 (95\% CI: $-1.68,-0.42)$ during activity at 6 hours after surgery and by 0.4 (95\% CI: $-0.8,-0.009)$ at 24 hours after surgery. This study was underpowered to detect such a small difference in pain scores between groups.

Intravenous lidocaine infusion has only shown benefit (in reducing pain, nausea, opioid consumption, bowel function, and reducing hospital stay) in patients following surgery of the bowel or gall bladder, ${ }^{8}$ with the exception of radical prostatectomy. ${ }^{16}$ The studies of intravenous lidocaine for patients undergoing abdominal hysterectomy, ${ }^{17}$ orthopedic surgery,${ }^{18}$ tonsillectomy, ${ }^{19}$ or coronary artery bypass ${ }^{20}$ could not identify any benefits of lidocaine. Based on the available evidence, it appears that the analgesic efficacy of lidocaine is dependent on the surgical procedure performed. We propose that the analgesic efficacy of lidocaine relates to the afferent (sensory) innervation of the manipulated tissues and the type of nociceptive pain associated with this innervation. 


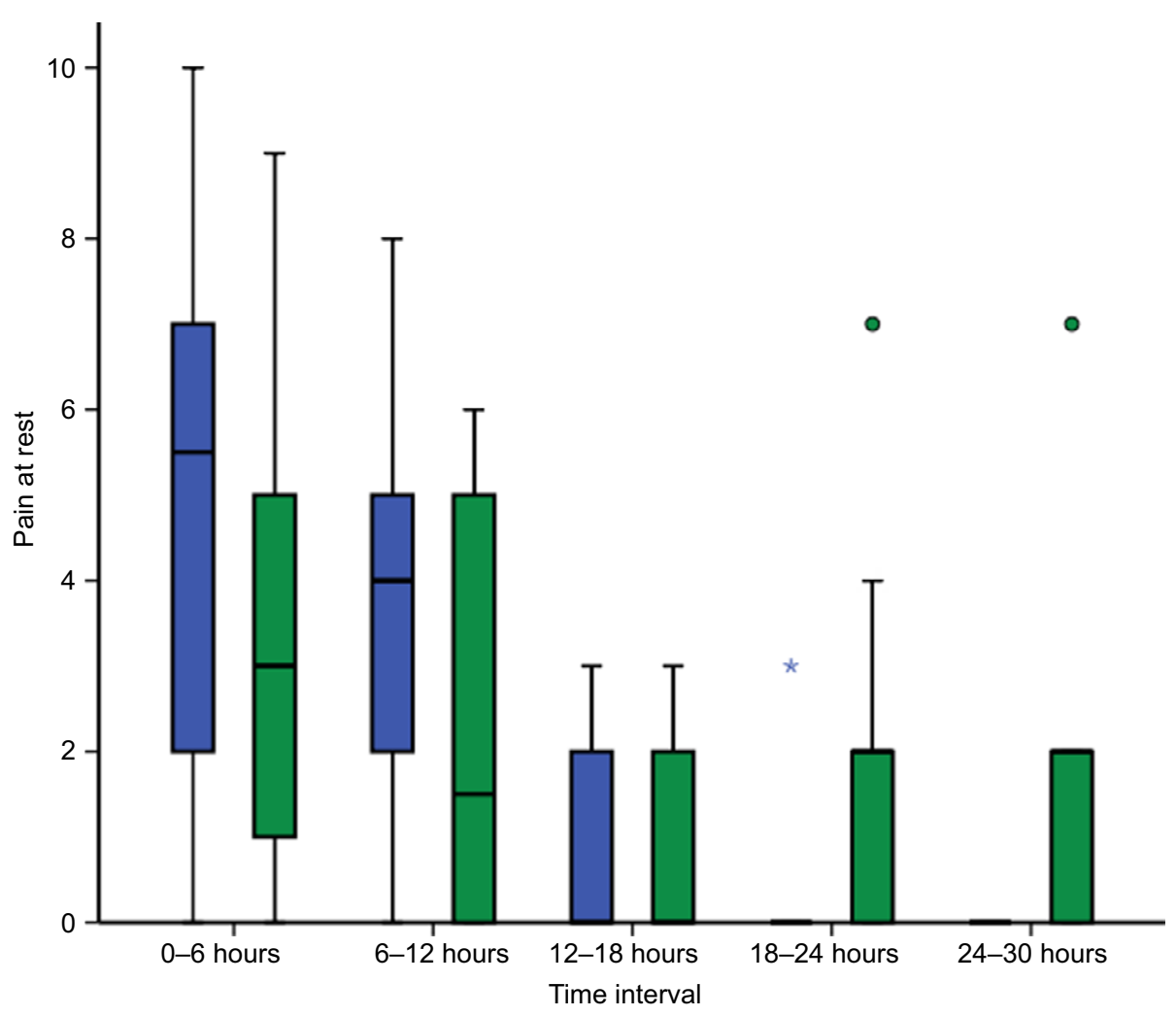

Figure 2 Box and whisker plot of pain scores at rest for lidocaine (blue) and control groups (green) at 6-hour intervals for 30 hours following laparoscopic fundoplication. Notes: $\odot$, outlier; $\star$, extreme outlier. Pain was assessed using an II-point numeric rating scale (NRS-I I). No significant difference between groups.

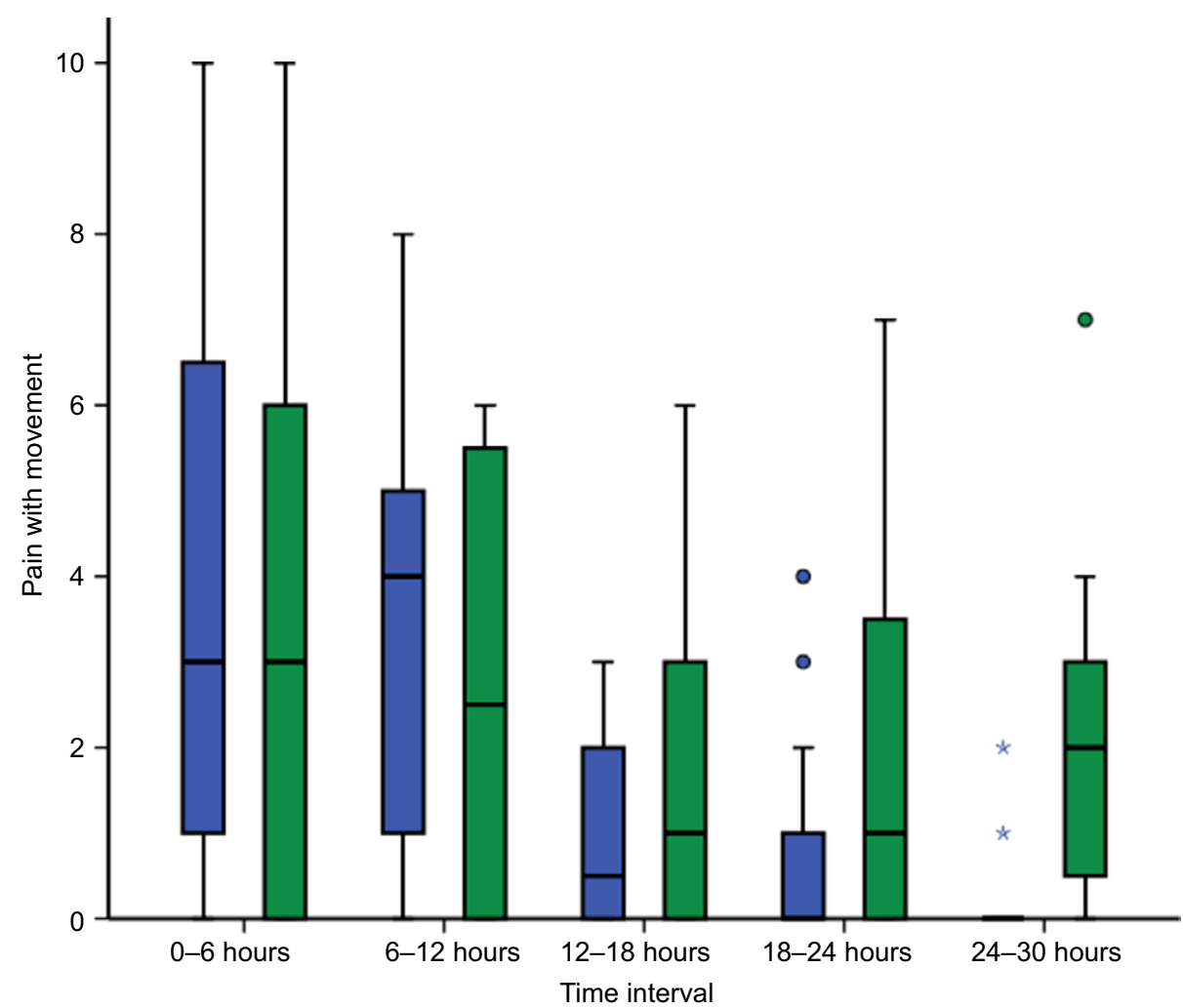

Figure 3 Box and whisker plot of pain scores with movement for lidocaine (blue) and control groups (green) at 6-hour intervals for 30 hours following laparoscopic fundoplication. Notes: $\odot$, outlier; $\star$, extreme outlier. Pain was assessed using an II-point numeric rating scale (NRS-II). No significant difference between groups. 


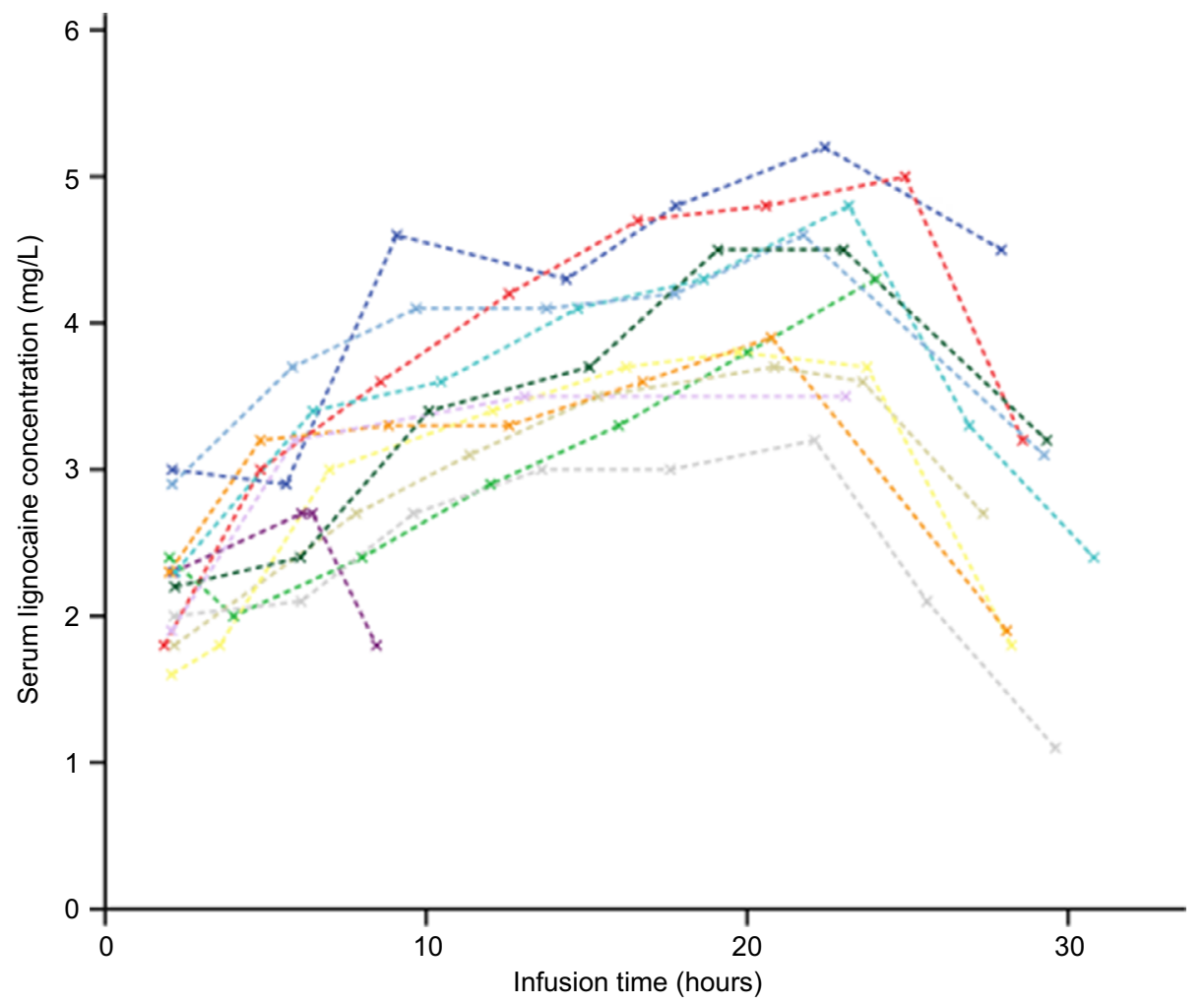

Figure 4 Serum concentration-time profile of lidocaine for each of the 12 patients receiving an intravenous lidocaine infusion ( $\mathrm{mg} / \mathrm{kg}$ loading dose, then $2 \mathrm{mg} / \mathrm{kg} / \mathrm{h}$ for 24 hours).

Note: Different colored lines represent each of the 12 patients.

It would seem that intravenous lidocaine has the potential to improve postoperative analgesia following abdominal surgical procedures associated with visceral pain or postoperative ileus. Laparoscopic fundoplication predominantly involves surgical manipulation of the diaphragm, which receives somatic sensory innervation via the phrenic nerve. The pneumoperitoneum and tissue combustion plume that are associated with laparoscopic surgery may also irritate the parietal peritoneum. Since there seems to be a greater proportion of somatic pain than visceral pain associated with laparoscopic fundoplication, this may explain why an analgesic benefit of lidocaine was not observed in this study.

This study had a higher incidence of lidocaine toxicity than previous studies. No correlation was found between the occurrence of adverse events and serum lidocaine concentration, however, this is not unusual, as local anesthetic toxicity is described as being insidious and often unexpected. ${ }^{21}$ Clinical signs and symptoms of lidocaine toxicity can occur below the much quoted "toxic threshold" of $5 \mathrm{mg} / \mathrm{L}$, as there are inter-individual differences between lidocaine serum concentration and its therapeutic or toxic effects. ${ }^{22}$ The greater number of adverse events seen in this study may be partially explained by the additional monitoring that the patients received. This unpredictability in safety may limit the clinical utility of intravenous lidocaine for postoperative analgesia.
The patients in this study were found to have greater serum lidocaine concentration at steady-state than was anticipated. The clearance of lidocaine in this study population was $7.9 \pm 1.32 \mathrm{~mL} / \mathrm{kg} / \mathrm{min}, \sim 20 \%$ less than the estimate used when formulating the dosage regimen. ${ }^{23}$ This study found that an infusion rate of $33 \mu \mathrm{g} / \mathrm{kg} / \mathrm{min}(2 \mathrm{mg} / \mathrm{kg} / \mathrm{h})$ resulted in mean steady state serum concentration of $4.1 \mathrm{mg} / \mathrm{L}$. An infusion rate of $26 \mu \mathrm{g} / \mathrm{kg} / \mathrm{min}(1.6 \mathrm{mg} / \mathrm{kg} / \mathrm{h})$ would have been required to result in the intended steady state serum concentration of $3.3 \mathrm{mg} / \mathrm{L}$ in this population.

The weakness of this study is the small sample size because the trial was stopped early on the grounds of futility. We acknowledge that this trial has a higher risk of Type II error and it cannot exclude the possibility of a true effect size less than the minimum effect size of interest stipulated in the power calculation, however, we believe that it would have been an improper use of resources and unacceptable risk to patient safety in continuing the trial given the improbability of achieving statistical significance even if the entire a priori sample size was recruited. The strengths of this study are the validity of the randomized controlled trial methodology, the integrity of data collection, and close monitoring of patients for adverse events. As such, this well-designed, albeit small, study could contribute to future systematic reviews and metaanalyses in this field. 
Further investigation is warranted to define the optimal dosage regimen for intravenous lidocaine to balance analgesic efficacy and patient safety. Only when an evidence-based dosage protocol has been derived should further studies be performed to identify which surgical procedures or patient populations may benefit from intravenous lidocaine.

This study does not support the use of intravenous lidocaine infusion for analgesia in patients undergoing laparoscopic fundoplication surgery. Intravenous lidocaine cannot be recommended for analgesia until a safe dosage regimen has been determined and the surgical procedures for which patients can benefit from intravenous lidocaine infusion have been identified.

\section{Acknowledgments}

The authors would like to acknowledge the staff at Sydney Adventist Hospital for their time and expertise that they dedicated to the project, Adventist HealthCare Limited for kindly facilitating the trial, and Sophie Liang for statistical support. Presented at the Australian Society of Anesthetists National Scientific Congress October 2014. This trial has not received sponsorship from the pharmaceutical industry.

\section{Disclosure}

GJD received research scholarships from the University of Sydney for his involvement in the project. The authors report no other conflicts of interest in this work.

\section{References}

1. Iqbal A, Kakarlapudi GV, Awad ZT, et al. Assessment of diaphragmatic stressors as risk factors for symptomatic failure of laparoscopic Nissen fundoplication. J Gastrointest Surg. 2006;10(1):12-21.

2. Taniguchi T, Shibata K, Yamamoto K, Mizukoshi Y, Kobayashi T. Effects of lidocaine administration on hemodynamics and cytokine responses to endotoxemia in rabbits. Crit Care Med. 2000;28(3):755-759.

3. Abram SE, Yaksh TL. Systemic lidocaine blocks nerve injury-induced hyperalgesia and nociceptor-driven spinal sensitization in the rat. Anesthesiology. 1994;80(2):383-391.

4. Sun Y, Li T, Wang N, Yun Y, Gan TJ. Perioperative systemic lidocaine for postoperative analgesia and recovery after abdominal surgery: a meta-analysis of randomized controlled trials. Dis Colon Rectum. 2012;55(11):1183-1194.

5. Mao J, Chen LL. Systemic lidocaine for neuropathic pain relief. Pain. 2000;87(1):7-17.
6. Marret E, Rolin M, Beaussier M, Bonnet F. Meta-analysis of intravenous lidocaine and postoperative recovery after abdominal surgery. Br J Surg. 2008;95(11):1331-1338.

7. Vigneault L, Turgeon AF, Cote D, et al. Perioperative intravenous lidocaine infusion for postoperative pain control: a meta-analysis of randomized controlled trials. Can J Anaesth. 2011;58(1):22-37.

8. McCarthy GC, Megalla SA, Habib AS. Impact of intravenous lidocaine infusion on postoperative analgesia and recovery from surgery: a systematic review of randomized controlled trials. Drugs. 2010;70(9):1149-1163.

9. Kranke P, Jokinen J, Pace NL, et al. Continuous intravenous perioperative lidocaine infusion for postoperative pain and recovery. Cochrane Database Syst Rev. 2015;70(9):1149-1163.

10. Breivik EK, Björnsson GA, Skovlund E. A comparison of Pain Rating Scales by sampling from clinical trial data. Clin J Pain. 2000;16(1):22-28.

11. Hjermstad MJ, Fayers PM, Haugen DF, et al; European Palliative Care Research Collaborative (EPCRC). Studies comparing Numerical Rating Scales, Verbal Rating Scales, and Visual Analogue Scales for assessment of pain intensity in adults: a systematic literature review. $J$ Pain Symptom Manage. 2011;41(6):1073-1093.

12. DeLoach LJ, Higgins MS, Caplan AB, et al. The visual analog scale in the immediate postoperative period: intrasubject variability and correlation with a numeric scale. Anesth Analg. 1998;86(1):102-106.

13. Farrar JT, Portenoy RK, Berlin JA, et al. Defining the clinically important difference in pain outcome measures. Pain. 2000;88(3):287-294.

14. Piantadosi S. Clinical Trials: A Methodologic Perspective. New York: John Wiley \& Sons; 2005.

15. Proschan MA, Lan KG, Wittes JT. Statistical Monitoring of Clinical Trials: A Unified Approach. New York: Springer Science \& Business Media; 2006.

16. Groudine SB, Fisher HA, Kaufman Jr RP, Patel MK, Wilkins LJ, Mehta SA, Lumb PD. Intravenous lidocaine speeds the return of bowel function, decreases postoperative pain, and shortens hospital stay in patients undergoing radical retropubic prostatectomy. Anesth Analg. 1998;86(2):235-239.

17. Bryson GL, Charapov I, Krolczyk G, Taljaard M, Reid D. Intravenous lidocaine does not reduce length of hospital stay following abdominal hysterectomy. Can J Anaesth. 2010;57(8):759-766.

18. Martin F, Cherif K, Gentili ME, et al. Lack of impact of intravenous lidocaine on analgesia, functional recovery, and nociceptive pain threshold after total hip arthroplasty. Anesthesiology. 2008;109(1):118-123.

19. Striebel HW, Klettke U. Is intravenous lidocaine infusion suitable for postoperative pain management? Schmerz. 1992;6(4):245-250.

20. Insler SR, O'Connor M, Samonte AF, Bazaral MG. Lidocaine and the inhibition of postoperative pain in coronary artery bypass patients. J Cardiothorac Vasc Anesth. 1995;9(5):541-546.

21. Davison R, Parker M, Atkinson Jr AJ. Excessive serum lidocaine levels during maintenance infusions: Mechanisms and prevention. Am Heart J. 1982;104(2, Part 1):203-208.

22. Collinsworth KA, Kalman SM, Harrison DC. The clinical pharmacology of lidocaine as an antiarrhythymic drug. Circulation. 1974;50(6):1217-1230.

23. Burm A, Van Kleef J, Vermeulen N, Olthof G, Breimer DD, Spierdijk J. Pharmacokinetics of lidocaine and bupivacaine following subarachnoid administration in surgical patients: simultaneous investigation of absorption and disposition kinetics using stable isotopes. Anesthesiology. 1988;69(4):584-592.
Local and Regional Anesthesia

\section{Publish your work in this journal}

Local and Regional Anesthesia is an international, peer-reviewed, open access journal publishing on the development, pharmacology, delivery and targeting and clinical use of local and regional anesthetics and analgesics. The journal is included in PubMed, and welcomes submitted papers covering original research, basic science, clinical studies,

\section{Dovepress}

reviews and evaluations, guidelines, expert opinion and commentary, case reports and extended reports. The manuscript management system is completely online and includes a very quick and fair peer-review system, which is all easy to use. Visit http://www.dovepress.com/ testimonials.php to read real quotes from published authors. 